\title{
Projéteis com Resistência do Ar Quadrática na Velocidade
}

The ballistic problem with square velocity air resistance

\author{
G. F. Leal Ferreira \\ guilherm@if.sc.usp.br \\ Instituto de Física de São Carlos, USP \\ Caixa Postal 369, 13.560-970, São Carlos, S.P.
}

Recebido em 2 de Maio 2001. Aceito em 26 de Junho 2001

\begin{abstract}
A solução aproximada do problema do movimento de projétil com resistência do ar quadrática na velocidade, obtida em 1719 por Johann Bernoulli, é comparada com soluções integradas numericamente a partir de uma primeira integral analítica da velocidade horizontal em termos do ângulo que o vetor velocidade faz com a horizontal, e é verificada ser adequada, pelo menos até ângulos de tiro de até $20^{\circ}$. Com ela resultados são obtidos em alguns casos práticos de interesse (tênis, futebol e outros) que dão elementos ao professor de física na área, para delimitar o que de prático tem o seu ensino em que a resistência do ar é ignorada.
\end{abstract}

Comparing approximate solution of the ballistic problem with air resistance proportional to the square of the velocity, obtained by Johann Bernoulli in 1719, with numerical ones obtained through an analytical solution expressing the horizontal component of the velocity in terms of the angle between the tangent to the trajectory and the horizontal axis, it is found it to be sufficiently good for shooting angles so high as $20^{\circ}$. Results of practical interest are then calculated (football and tennis game among other cases) which may give elements to the physics teacher in this area, of knowing how good is his teaching usually ignoring air resistance.

\section{Introdução}

O estudo do movimento dos projéteis (ideal) no ensino de Mecânica é importante por ser o primeiro caso - fora talvez o do movimento circular uniforme -, de composição de movimentos no plano. Entretanto, em que situações pode este estudo idealizado ser aplicado à prática em que o ar impõe resistência ao mesmo? No que apresentamos a seguir, procuramos familiarizar um pouco o professor com o problema da resistência do ar de forma a lhe permitir delimitar o alcance dos seus ensinamentos. Para velocidades da ordem de $\mathrm{m} / \mathrm{s}$, até suficientemente inferiores à velocidade do som, a resistência do ar é bem representada como proporcional ao quadrado da velocidade, estabelecida por Newton [1]. Para projéteis propriamente ditos (bala de fuzil ou de canhão), a resistência é mais complicada pela emissão de ondas e, por isso, nos restringiremos ao caso sub-sônico mais simples. Por outro lado, o caso da resistência do ar proporcional à velocidade, embora tratável analiticamente, não é realista.

Johann Bernoulli (daqui em diante J. Bernoulli; uma brevíssima nota biográfica sobre a famosa família Bernoulli é feita na seção 6) em 1719 obteve uma solução aproximada do problema [2], válida para trajetórias achatadas, isto é, com ângulo de tiro pequeno. Quão grande pode este ser, a solução de J. Bernoulli mantendo-se razoavelmente boa? Para responder a esta pergunta deve-se dispor de uma solução melhor, de preferência 'exata'. Acontece que se pode obter um resultado analítico para a velocidade horizontal do projétil no caso em questão, em função do ângulo que o vetor velocidade faz com a horizontal. Como hoje dispomos das facilidades de integração numérica proporcionada pelos computadores, a integração para se obter os espaços percorridos pode ser obtida logo. Com isto verificamos que para ângulos de tiro de $20^{\circ}$, a solução de J. Bernoulli é boa e, possivelmente, o é mesmo para ângulos maiores.

A análise do problema é muito simplificada pelo fato de se poder aferir a 'condutância' do meio ao projétil (condutância como inverso de resistência) em unidades de comprimento - o comprimento de condutância, $x_{c}$-, e daí se poder definir distâncias por meio de $x_{c}$, além de também por meio da outra unidade natural do problema, o alcance ideal $x_{0}$. Isto nos permitirá estudar a variação da relação alcance real/alcance ideal através do parâmetro $x_{0} / x_{c}$. 
As expressões teóricas, saidas de tratamentos conhecidos na literatura [2-4] e usadas na análise a seguir, estão deduzidas nos Apêndices, I e II. Na seção II adaptamos aquelas expressões aos nossos fins.

\section{Preparação}

Os principais resultados da análise teórica são a expressão da velocidade do projétil vem função do ângulo que o seu vetor velocidade faz com a horizontal (ver Fig.1, tirada de [1]),

$$
v^{2}(\theta)=\frac{v_{0}^{2} \cos ^{2} \alpha}{\left[1-\frac{2 v_{0}^{2} k \cos ^{2} \alpha}{g}(\xi(\theta)-\xi(\alpha))\right] \cos ^{2} \theta}
$$

resultado tirado da Eq.A11, e as equações determinando os deslocamentos horizontal, $x$, e vertical, $y$, Eqs. A13-A14,

$$
\begin{gathered}
x(\theta)=-\int_{\alpha}^{\theta} \frac{v^{2}(\theta) d \theta}{g}, \\
y(\theta)=-\int_{\alpha}^{\theta} \frac{v^{2}(\theta) \operatorname{tg} \theta d \theta}{g} .
\end{gathered}
$$

Outro resultado, este no Apêndice II, é a aproximação de J. Bernoulli (1719) [2], válida para trajetórias achatadas, Eq. A19,

$$
y=x \operatorname{tg} \alpha-\frac{x^{2} g}{2 v_{0}^{2} \cos ^{2} \alpha} F(z)
$$

com, Eq. A20,

$$
z=\frac{2 k x}{m} .
$$

Nas Eqs. 1 e $4, \alpha$ e $v_{0}$ são o ângulo de tiro e a velocidade inicial e $g$ a aceleração da gravidade. Na Eq. 5 $m$ é a massa e $k$ é o coeficiente relacionando a força de resistência $R$ e o quadrado da velocidade,

$$
R=k v^{2},
$$

o qual pode ser analisado nos seguintes termos:

$$
k=\frac{C A}{2} \rho_{a r}
$$

sendo $\rho_{a r}$ a densidade do ar $\left(\simeq 1,3 \mathrm{~kg} / \mathrm{m}^{3}\right), A$ a seção do projétil perpendicular à trajetória e $C$ um fator de forma que para uma esfera lisa é $\simeq 0,4[5]$. A dependência quadrática da resistência do ar foi deduzida por Newton, pelo momento na unidade de tempo comunicado pelo projétil ao ar [1].
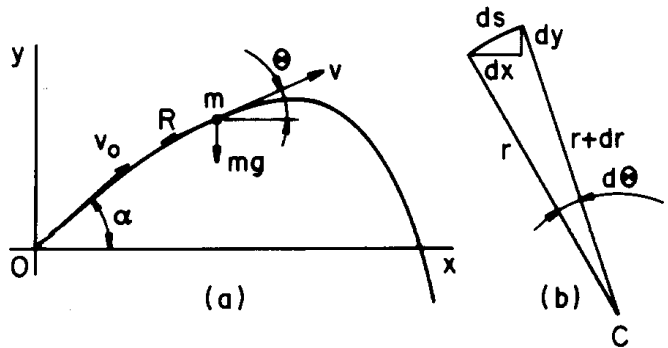

Figura 1: a) Esboço da trajetória de um projétil de massa $m$, lançado com ângulo de tiro $\alpha$ e velocida inicial $v_{0}, \theta$ e $v$ sendo o ângulo com a horizontal num ponto genérico da mesma. b) esboço do elemento de trajetória ds, sendo $r$ o raio de curvatura.

A função $\xi(\beta)$ na Eq. 1 e $F(z)$ na Eq. 4 são, respectivamente, A12 e A21,

$$
\begin{gathered}
\xi(\beta)=0,5\left[\frac{\operatorname{sen} \beta}{\cos ^{2} \beta}+0,5 \ln \frac{1+\operatorname{sen} \beta}{1-\operatorname{sen} \beta}\right], \\
F(z)=\frac{e^{z}-1-z}{0,5 z^{2}} .
\end{gathered}
$$

Para $z$, Eq.5, igual a zero, $F(0)=1$ e a trajetória da Eq.4 torna-se ideal. Vê-se que $m / k$ na Eq.5 tem a dimensão de comprimento, o que permite definir o comprimento de condutância $x_{c}=m / k$.

Assim, para $k=0, x_{c} \rightarrow \infty$. Maior resistência resulta em menor $x_{c}$. Uma outra unidade natural do problema é o alcance horizontal ideal $x_{0}$, que pela Eq. 2 é

$$
x_{0}=\frac{v_{0}^{2} \operatorname{sen} 2 \alpha}{g} .
$$

Com o valor de $x_{0} / 2$ na equação da trajetória ideal, encontra-se o valor da altura máxima, $y_{m}$,

$$
y_{m}=\frac{x_{0} \operatorname{tg} \alpha}{4}
$$

que permite aproximadamente estimar como achatada é a trajetória, $y_{m} / x_{0}<<1$.

Através da Eq.10, podemos substituir $v_{0}^{2} / g$ nas Eqs. 2 e 3 em termos de $x_{0}$, obtendo-se

$$
\begin{aligned}
& \frac{x(\theta)}{x_{0}}=-0,5 \cot \alpha \int_{\alpha}^{\theta} \frac{d \theta}{\left[1-\frac{x_{0}}{x_{c}}(\cot g \alpha)(\xi(\theta)-\xi(\alpha))\right] \cos ^{2} \theta} \\
& \frac{y(\theta)}{x_{0}}=-0,5 \cot \alpha \int_{\alpha}^{\theta} \frac{\operatorname{tg} \theta d \theta}{\left[1-\frac{x_{0}}{x_{c}}(\cot \alpha)(\xi(\theta)-\xi(\alpha))\right] \cos ^{2} \theta}
\end{aligned}
$$


em que 'cot' significa cotangente.

\section{O alcance horizontal}

Podemos estimar a importância da resistência do ar no movimento do projétil estudando como varia a relação entre o alcance horizontal real, $x_{r}$, e o alcance horizontal ideal, $x_{0}$, com a resistência. Para isto, integram-se as Eqs.12 e 13 até que a última das duas se anule (ou na prática, troque de sinal). Isto ocorre para um valor de $\theta, \theta_{m}=-\gamma \alpha$, negativo, $\operatorname{com} \gamma>1$ e tanto maior quanto menor for $x_{c}$. Na Fig. 2 mostramos $x_{r} / x_{0}$ como função de $x_{0} / x_{c}$, círculos cheios, e na Fig. $3, \gamma$ como função do mesmo $x_{0} / x_{c}$, para $\alpha=20^{\circ}$. Na verdade, $x_{r} / x_{0}$ e $\gamma$ tem dependência fraca $\operatorname{com} \alpha, \operatorname{com} \alpha$ pequeno. Esta independência de $x_{r} / x_{0} \operatorname{com} \alpha$ pode ser suspeitada pela relação válida na aproximação J. Bernoulli, Eq.4, que em função de $x_{0}$ e $x_{c}$ é

$$
\frac{y}{x_{0}}=\frac{x}{x_{0}} \operatorname{tg} \alpha\left(1-\frac{x}{x_{0}} F\left(\frac{2 x}{x_{c}}\right)\right),
$$

da qual se tira

$$
\frac{x_{r}}{x_{0}}=\frac{1}{F\left(\frac{2 x_{r}}{x_{c}}\right)}
$$

independente de $\alpha$ e que permite a obtenção da relação entre $x_{r} / x_{0}$ e $x_{0} / x_{c}$. Para isto, o valor de $u=$ $2 x_{r} / x_{c}$ que satisfaz a Eq.15 é escrito como $u=$ 2. $\left(x_{r} / x_{0}\right) \cdot\left(x_{0} / x_{c}\right)$ e daí calcula-se $x_{0} / x_{c}$ para cada $x_{r} / x_{c}$. Na Fig. 2 também mostramos este resultado da aproximação de J. Bernouilli, por círculos vazados. Vemos que o acordo entre esta e a solução 'exata' é bastante razoável, fornecendo valores de $x_{r} / x_{0}$ um pouco superiores do que a exata. Pela Fig. 2 vemos que para $x_{0} / x_{c}$ igual a 0,5 e $1, x_{r} / x_{0}$ é cerca de 0,76 e 0,62 , aproximadamente. O primeiro valor, $x_{0} / x_{c}=0,5$, vai ser considerado, com certa arbitrariedade, como aquele para o qual a resistência do ar começa a ser importante.

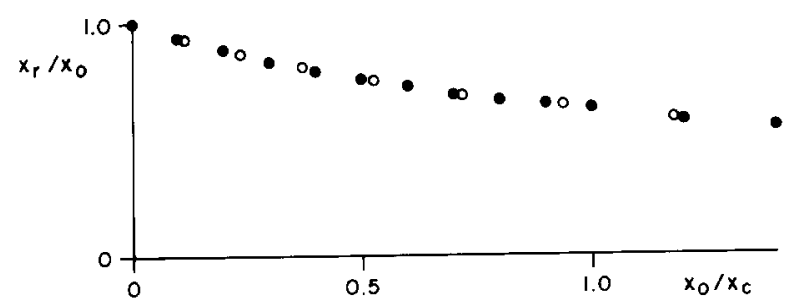

Figura 2: Alcance real/alcance ideal, $x_{r} / x_{0}$, em função do parâmetro alcance ideal/comprimento de condutância, $x_{0} / x_{c}$, calculado a partir das Eqs. 12 e 13, ver texto, em círculos cheios, e calculado com a aproximação J. Bernoulli, Eq. 15, em círculos vazados, para $\alpha=20^{\circ}$.

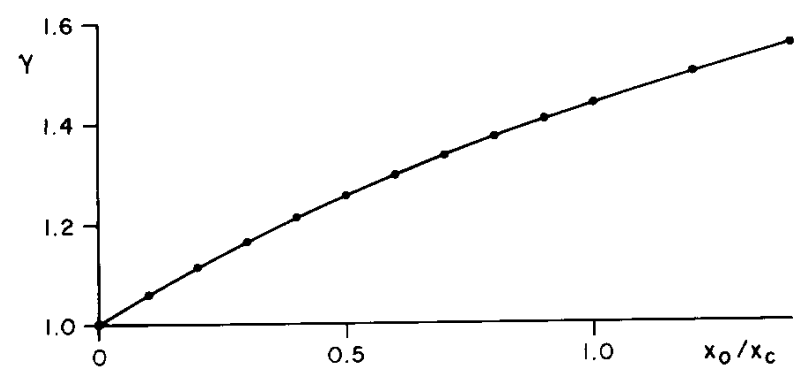

Figura 3: $\gamma$ como função de $x_{0} / x_{c}$ obtido das Eq.12 e 13 . $-\gamma \alpha$ é o ângulo com que o projétil atinge o eixo horizontal $\alpha=20^{\circ}$.

\section{Trajetórias J. Bernoulli}

O bom resultado proporcionado pela aproximação J. Bernoulli nos anima a obter dela, Eq. 14, trajetórias, de forma bem mais simples do que pelas exatas, Eqs. 12 e 13. Escrevendo a Eq. 14 como

$$
\frac{y}{x_{0}}=\frac{x}{x_{0}} \operatorname{tg} \alpha\left(1-\frac{x}{x_{0}} F\left(2 \frac{x_{0}}{x_{c}} \frac{x}{x_{0}}\right)\right),
$$

mostramos na Fig. 4 (note a diferença entre as escalas vertical e horizontal) trajetórias calculadas para $x_{0} / x_{c}$ igual a $0,0,23,0,53$ e 0,93 e $\alpha=20^{\circ}$. Vê-se que a altura máxima ideal, $y_{m}$, Eq. 11 , é só cerca de $9 \%$ do alcance horizontal ideal, $x_{0}$, no presente caso, justificando o epíteto de 'achatada'.

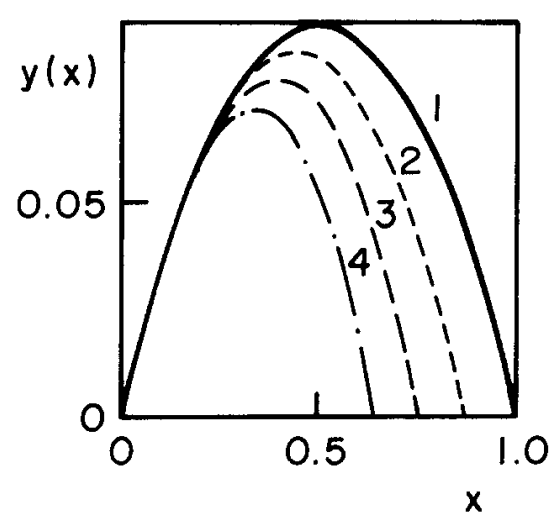

Figura 4: Trajetórias J. Bernoulli, Eq.14, para $x_{0} / x_{c}=0$, $0,23,0,53$ e 0,93 e $\alpha=20^{\circ}$.

\section{Alguns casos práticos}

Adotamos no final da seção 3 o critério segundo o qual a resistência do ar se torna importante quando $x_{0} / x_{c}$ é 0,5 . Vamos agora tomar alguns projéteis familiares esféricos para aquilatarmos suas performances frente à resistência do ar. Antes, porém, calculemos o valor de $x_{c}$, dado pela Eq. 7 , com $C=0,4, \rho_{a r}=1,3 \mathrm{~kg} / \mathrm{m}^{3}$, sendo $D$ o diâmetro, através de 


$$
x_{c}=\frac{8 m}{(0,4) \pi D^{2}(1,3)}=\frac{4,9 m}{D^{2}}
$$

a) Jogo de tenis. A bola de tenis 'pesa' cerca de 2 onças e tem o diâmetro de 2,5 polegadas [6]. 1 onça $=28,35 \mathrm{~g}$ e 1 polegada $=2,54 \mathrm{~cm}$. Obteríamos $x_{c}=69 \mathrm{~m}$, mas como a bola de tenis é bastante rugosa, $C$ deve ser maior, vamos dizer 0,6. Com isto $x_{c}$ é cerca de $46 \mathrm{~m}$. Como a quadra de tenis para jogo de simples tem 42 pés [6], ou seja $\approx 14 \mathrm{~m}$ pode-se dizer que mesmo para lances longos a resistência do ar não é determinante no jogo de tenis.

b) Jogo de futebol: a bola de futebol tem cerca de $71 \mathrm{~cm}$ de perímetro e $456 \mathrm{~g}$ de 'peso' [7]. Usando estes valores na Eq. 16 obtemos $x_{c}=44 \mathrm{~m}$. portanto para chutes de $22 \mathrm{~m}$ de alcance ideal, correspondendo à velocidade inicial de $18,3 \mathrm{~m} / \mathrm{s}$, a resistência do ar é importante.

Estudemos agora os corpos massivos (não ocos). Para estes corpos, a Eq. 17 pode ser expressa em termos da densidade $\rho$ e do diâmetro $D$ do projétil como

$$
x_{c}=2,56 \rho D
$$

mostrando que a resistência tende a ser mais importante para menores $D$. Tomemos um sólido bem leve, por exemplo o isopor (poli-estireno expandido) cuja densidade no ar foi por nós estimada em $0,033 \mathrm{~g} / \mathrm{cm}^{3}$ ou 33 $\mathrm{kg} / \mathrm{m}^{3}$. Para $D=4 \mathrm{~cm}=0,04 \mathrm{~m}$, obtemos para $x_{c}$, Eq. 18, 3,8 m. Portanto, para o projétil de isopor a resistência do ar será importante para alcances.superiores a 1,9 m, correspondendo a uma velocidade inicial de 5,4 $\mathrm{m} / \mathrm{s}$, ou seja, praticamente $20 \mathrm{~km} / \mathrm{h}$ (o isopor parece ser o material indicado para o estudo da influência da resistência do ar para velocidades de $\mathrm{m} / \mathrm{s}$ ). Com a densidade usual, isto é da ordem de $\mathrm{g} / \mathrm{cm}^{3}, x_{c}$ seria cerca de 30 vezes maior e a resistência do ar não será usualmente importante. Nesta situação se enquadram as experiências que são realizadas no laboratório em aulas de Mecânica. Mas se pequenos desvios do comportamento ideal quiserem ser tomados em consideração a seguinte aproximação é válida até $x_{0}=0,1$, extraida da Fig.2,

$$
\frac{x_{r}}{x_{0}} \cong 1-0,61 \frac{x_{0}}{x_{c}}
$$

\section{Nota bibliográfica brevíssima}

Vários Bernoulli's, ao longo de gerações durante os séculos 17 e 18, se destacaram em estudos de Matemática, Física e Teoria das Probabilidades [6]. Os dois primeiros foram Jakob, mais velho, e Johann, irmãos, mas que nem por isso viveram sempre em harmonia. O Bernoulli do teorema de Bernoulli da Hidrodinâmica é Daniel, filho de Johann.

\section{Agradecimentos}

O autor agradece a bolsa de produtividade do C.N.Pq e ao árbitro deste por algumas de suas sugestões. E à colega Profa. Mariangela T. de Figueiredo pela leitura atenta.

\section{Apêndice I - Resultado Analítico}

Seguiremos de perto a Ref. [2], havendo porém um pequeno erro de sinal na sua Eq. 74, Cap.1, correspondendo a nossa Eq. 11 abaixo. A Ref. [3] também apresenta este estudo, porém com erro mais importante nessa mesma expressão, Eq. 8.8, Cap. IV. Coincidência. A Ref. [4] também apresenta tratamento do problema geral da resistência dependente da velocidade, mas, como dissemos, seguiremos a Ref. [2]. Naturalmente que complicações outras como a dependência da resistência do ar, com a altura do projétil, através da densidade do ar variável, não serão consideradas aquí.

Preliminarmente, pela definição de raio de curvatura $r$ temos, ver Fig.1.a e 1.b, que

$$
\frac{1}{r}=\frac{d \theta}{d s}
$$

em que $\theta$ é o ângulo que o vetor velocidade faz com a horizontal e $d s$ é o elemento de arco. Por outro lado, também sabemos que

$$
\frac{1}{r}=\frac{\frac{-d^{2} y}{d x^{2}}}{\left[1+\left(\frac{d y}{d x}\right)^{2}\right]^{3 / 2}}
$$

sendo $x$ e $y$ as coordenadas cartesianas dos pontos da curva. Vê-se facilmente que podemos pôr

$$
\frac{1}{r}=-\frac{d^{2} y}{d x^{2}} \cos ^{3} \theta \text {. }
$$

A aceleração centrípeta é $v^{2} / r$, sendo $v$ a velocidade, ou pela Eq. A1

$$
\frac{v^{2}}{r}=-v^{2} \frac{d \theta}{d s}
$$

e usando a equação da força centrípeta, com $m$ a massa, ver Fig. 1a,

$$
\frac{m v^{2}}{r}=-m v^{2} \frac{d \theta}{d s}=m g \cos \theta
$$

resultando

$$
v^{2} \frac{d \theta}{d s}=-g \cos \theta
$$

ou ainda, como $v=d s / d t$,

$$
\frac{d \theta}{d t}=-\frac{g \cos \theta}{v}
$$


É interessante que uma primeira integração será possível através da variável $v_{x}=v \cos \theta$.

Começando por escrever a 2a lei na direção horizontal

$$
m \frac{d v_{x}}{d t}=-R \cos \theta=-k v^{2} \cos \theta
$$

sendo $R=k v^{2}$ a força de resistência, e substituindo $d t$ da Eq.7, temos

$$
\frac{d v_{x}}{d \theta}=-\frac{k v^{3}}{m g}
$$

e multiplicando e dividindo o lado direito por $\cos ^{3} \theta$

$$
\frac{d v_{x}}{v_{x}^{3}}=-\frac{k d \theta}{m \cos ^{3} \theta}
$$

que é uma equação diferencial integrável em $v_{x}$ e $\theta$, obtendo-se

$$
v_{x}^{2}=v^{2} \cos ^{2} \theta=\frac{v_{0}^{2} \cos ^{2} \alpha}{1-\frac{2 k v_{0}^{2} \cos ^{2} \alpha}{m g}(\xi(\theta)-\xi(\alpha))}
$$

em que

$$
\xi(\beta)=\int_{0}^{\beta} \frac{d \theta}{\cos ^{3} \theta}=0,5\left(\frac{\operatorname{sen} \theta}{\cos ^{2} \theta}+0,5 \ln \frac{1+\operatorname{sen} \theta}{1-\operatorname{sen} \theta}\right),
$$

sendo $\alpha$ e $v_{0}$ o ângulo de tiro e velocidade iniciais, respectivamente. Foi por meio da tabela de integrais da Ref. [6] que detetamos os erros mencionados. Com $v_{x}$ na Eq.11, $d x$ e $d y$ podem ser obtidos de $d x=d s \cos \theta$ e $d y=d s \operatorname{sen} \theta$ e usando-se a Eq.6 tem-se

$$
\begin{gathered}
x(\theta)=-\int_{\alpha}^{\theta} \frac{v^{2} d \theta}{g}, \\
y(\theta)=-\int_{\alpha}^{\theta} \frac{v^{2} \operatorname{tg} \theta d \theta}{g} .
\end{gathered}
$$

\section{Apêndice II - Aproximação de J. Bernoulli}

A dedução da aproximação de J. Bernoulli começa considerando as Eqs. A9 e A6 obtém-se

$$
\frac{d v_{x}}{v_{x}}=\frac{k v^{2} d \theta}{m g \cos \theta}=-\frac{k d s}{m}
$$

equação que pode ser integrada

$$
v_{x}=v_{x 0} e^{-k s / m}
$$

sendo $v_{x 0}$ a componente $x$ da velocidade inicial. Para trajetórias achatadas $s \approx x$, de maneira que

$$
v_{x}=\dot{x} \cong v_{x 0} e^{-k x / m}=\dot{x}_{0} e^{-k x / m}
$$

e pelas Eqs. A3, A5 e A17 chega-se a, já com o sinal de igualdade, $(\cos \theta \approx 1)$

$$
\frac{d^{2} y}{d x^{2}}=-\frac{g}{\dot{x}^{2}}=-\frac{g e^{2 k x / m}}{\dot{x}_{0}^{2}}
$$

equação esta que integrada com $y=0$ para $x=0$ e $d y / d x)_{0}=\operatorname{tg} \alpha$ dá

$$
y(x)=x t g \alpha-\frac{g x^{2}}{2 v_{0}^{2} \cos ^{2} \alpha} F(z)
$$

com

$$
\begin{gathered}
z=\frac{2 k x}{m} \\
F(z)=\frac{e^{z}-1-z}{0,5 z^{2}}
\end{gathered}
$$

Note que para $z=0$, meio sem resistência, $F(0)=1$.

\section{References}

[1] S. Timoshenko e D. H. Young, Dinamica Avanzada, Libreria Hachette, Buenos Aires, Cap.1, seção 4.

[2] O mesmo, Cap.1, seção 12.

[3] T.von Kármán e Maurice Biot, Mathematical Methodas in Engineering, McGraw-Hill Book Co., N.York, 1940, Cap. IV, seção 8.

[4] John L. Synge e Byron A. Griffith, Principles of Mechanics, MCGraw-Hill Book Co., 1959, pag. 142.

[5] Victor L. Streeter, Mecânica dos Fluidos, McGraw-Hill do Brasil, 1974, pg.261.

[6] Encyclopaedia Britanica, ed.1969.

[7] Leopoldo Sant'Ana, Regras de Futebol Ilustradas, Cia. Brasil Editora, São Paulo, 1968.

[8] Handbook of Chemistry and Physics, $55^{\text {th }}$ Edition, 19741975, A111. 